\title{
理论教学与实践教学互相渗透的物理化学教学模式探讨
}

王女“，赵勇，刘兆阅，田东亮

北京航空航天大学化学学院, 北京 100191

摘要: 针对目前存在的物理化学课程中理论教学与实践教学脱节的问题, 结合自身教学改革和探索, 提出了理论与 实践互相渗透的教学模式。实践证明, 有意识地将理论教学与实践教学结合起来, 可以激发学生学习的主观能动性, 深化知识点理解, 对提升学生分析问题能力、解决问题能力, 以及创新能力都有良好的效果。

关键词: 理论教学; 实验教学; 物理化学

中图分类号: G64; O6

\section{Discussion on Teaching Modes of Physical Chemistry between Theoretical Teaching and Laboratory Course}

Nü Wang *, Yong Zhao, Zhaoyue Liu, Dongliang Tian

School of Chemistry, Beihang University, Beijing 100191, P. R. China.

Abstract: Aiming at the problem of the disconnection between theoretical teaching and laboratory course in physical chemistry, we propose teaching mode between theoretical teaching and laboratory course based on the author's teaching reform and exploration. It has been proved that combination of theoretical and laboratory teaching could stimulate students' subjective initiative in learning, and deepen the understanding of knowledge points. It will significantly improve students' ability to analyze problems, solve problems and innovate ability.

Key Words: Theoretical teaching; Laboratory course; Physical chemistry

物理化学是一门从物理学的角度分析物质体系化学行为的原理、规律和方法的学科, 是近代化 学的原理和根基。物理化学课程概念性、理论性、系统性和逻辑性都很强, 需要无机化学、高等数 学、物理学的前期知识支撑, 概念抽象、公式推导复杂, 且相互关联, 因此, 初学者往往会产生畏 惧。为了激发学生的学习兴趣和热情, 引导学生以正确的方法学习并掌握物理化学课程内容, 广大 的物理化学教师们需要在教学方法、教学内容、教学模式等方面进行全方位的改革和探索。物理化 学实验是继无机化学实验、有机化学实验、分析化学实验后需要综合运用物理和化学的原理、技术、 仪器, 以及数学运算工具, 开设的一门培养学生理论性、实践性、研究性等综合能力的课程。为了 提高教学质量, 改善教学效果, 在近几年的物理化学课程教学过程中, 本人以理论教学和实验教学 相结合为目标, 通过理论教学和实验教学互相渗透的教学模式, 在物理化学教学过程中进行了一些 新的教学手段及方法探索, 引导学生在学习过程中注重理论知识的实际应用, 掌握获取知识的实验 方法, 注重实验结果的讨论和分析, 与科学前沿相结合, 充分调动学生的求知欲和自主学习的积极 性, 力图使理论知识和实践教学内容相辅相成, 相互促进, 从而提高教学效果, 培养学生综合解决

收稿: 2019-07-02; 录用: 2019-09-12; 网络发表: 2019-09-25

“通讯作者, Email: wangn@buaa.edu.cn

基金资助: 北京航空航天大学教改项目 
问题的能力。

\section{1 物理化学课堂理论教学与实验教学中存在的主要问题}

\section{1 理论教学内容与实验脱节}

物理化学是一门抽象的、公式繁多的课程, 学好这门课的基础是充分理解其中的知识点, 并利 用知识点结合具体条件应用理论分析解决较为简单的化学热力学及动力学问题。物理化学实验恰恰 是需要利用课堂上所讲的理论知识, 结合仪器等培养学生实验操作、观察、思维、数据处理、分析 问题、解决问题的综合能力。然而, 在很长一段时间的教学过程中, 物理化学理论教学内容与实验 教学是脱节的。理论教师和实验教师往往分别由不同的教师担任, 缺乏沟通。理论课教师注重知识 点讲解、公式推导和习题运算, 而忽视了物理化学实验课的教学内容。而实验课教师的教学重点在 于仪器的使用、实验操作、实验注意事项、如何处理实验数据等能让学生顺利完成实验的环节上。 在这样的教学模式下, 学生经常机械地完成了实验课程, 却没有深入考虑其中所对应的知识点和应 该掌握的实验原理, 没有达到物理化学实验课的教学目的, 也不利于培养学生学习物理化学知识的 积极性 ${ }^{[1-3]}$ 。

\section{2 实验课程排课时间与理论脱节}

实验课程与理论课程脱节, 不仅表现在实验内容上, 还体现在物理化学实验的时间设置和安排 上。与无机化学实验、有机化学实验、分析化学实验不同, 物理化学实验中所用的设备仪器往往更 为复杂, 无法做到全班学生单人单套。因此物理化学实验往往采取分组, 几个实验循环进行的模式 开展, 所以造成课堂教学与实验进度不同步。有的时候会出现, 实验开了, 而对应的知识点还没讲 到的情况。学生虽然可以参考实验讲义, “照猫画虎” 地完成实验, 但是并没有消化其中的知识点。

\section{3 理论课、实验课与科研割裂}

由于仪器设备更新速度较慢, 存在着实验内容单一、陈旧等问题, 实验内容和操作方法与目前 高速发展的化学仪器和表征手段存在着明显脱节。由于化学、材料、环境、生物等专业的学生很大 一部分将来会考取研究生继续深造或者从事相关的专业工作, 而目前实验课上所用的仪器设备、实 验方法技术与将来要用到的仪器设备差别较大, 这会大大影响学生们上实验课的积极性。一成不变 的实验内容、单一陈旧的实验设备、简单的验证性实验, 不利于学生创新能力和主动性的培养。而 在真正的生产或者是科学研究过程中, 不确定性和意想不到的问题往往是经常发生的, 如何处理这 些问题，分析原因、解决问题才是学生和老师真正应该思考的问题。

\section{2 理论教学与实践相互渗透的改革措施}

\section{1 理论授课与实验内容有机结合}

在理论授课过程中渗透实验, 理论课教师应充分了解每学期的实验设置和安排, 在理论课的教 学中适当穿插物理化学实验的原理讲解 ${ }^{[4-6]}$ 。由于物理化学理论课程中公式推导较多, 内容较为枯 燥, 如果单纯从物理量引入, 学生理解有一定难度, 因此, 在一些物理量的引入过程中, 教师采用 由实验引入的方法, 首先讲解物理量的测试方法及实验, 再引入物理量及理论公式, 由浅入深, 循 序渐进, 让学生深入理解物理化学课程的内涵, 掌握化学过程的规律, 由已知量或易测量获得未知 量或难测量。例如, 在相平衡二元双液系液相完全互溶、定压条件下 $T-x$ 相图讲解过程中, 自然地 将物理化学实验 “双液体系沸点-成分图的绘制” 引入其中。如图 1 所示, 测定几组不同含量丙醇-乙 醇(A-B 两组分)混合溶液的沸点及该浓度下气液平衡时气相、液相样品的折光率, 通过折光率对组 成的标准曲线获得气液平衡时气相 $\left(x_{\mathrm{G}}\right)$ 和液相 $\left(x_{\mathrm{L}}\right)$ 组成。将不同浓度混合溶液气液平衡时气相组成的 点连接起来即为气相线, 液相组成的点连接起来即为液相线, 就得到了丙醇-乙醇混合溶液的相图。 由于目前大部分高校的物理化学实验课安排采取循环制, 在讲到相平衡一章时有部分学生已经完成 了 “双液体系沸点-成分图的绘制”这一实验。在二元双液系液相完全互溶、定压条件 $T-x$ 相图的引入 
过程中, 请做过实验的学生描述所绘制的丙醇-乙醇相图的实验方法和相图形状, 在学生回答问题的 过程中, 对于实验原理和注意事项, 要稍加提醒, 并且将相图的含义结合实验进行深入讲解。进而 讲解相图中点、线、面的含义。而且相图上沸点与液相组成和气相组成对应关系的描点绘图过程, 将对二元相图中液液两相共存、沸点和组成的一一对应关系这一难点的理解非常有帮助。又如: 在 “化学动力学” 一章中, 讲到简单级数反应动力学方程, 由浓度和时间的关系可以获得简单级数反 应的动力学方程, 提问学生在具体的实验过程中, 是否思考过如何测量反应物或者生成物的浓度, 在真实的化学反应中, 反应物或者是生成物的浓度是不断变化的, 要想直接测量其浓度最直接的方 法是冻结反应, 这在实际的实验中是很难实现的。我们在实验的过程中往往采用的是间接测量的方 法, 通过连续对某选定物理量进行监测, 如旋光度、折射率、电导率、粘度, 或现代谱仪监测与浓 度有定量关系的物理量的变化, 间接获得浓度和时间的关系, 从而得到动力学曲线。课堂上提醒学 生在后续的实验课程中会做 “蔗糖水解反应速率的测定” , 这一实验就是通过测量旋光度来间接获 得动力学曲线的。通过课堂上设计这样的教学方法, 既可以引起已经做过该实验的部分学生的注意, 加深他们对实验原理的理解, 又可以增强未做过该实验的学生做实验的积极性; 他们会利用已经学 过的知识点, 更加深入地掌握实验原理。因此, 理论课教师适当地将实验内容引入课堂, 对提高学 生学习兴趣和加深学生对知识点的理解都是非常必要的。

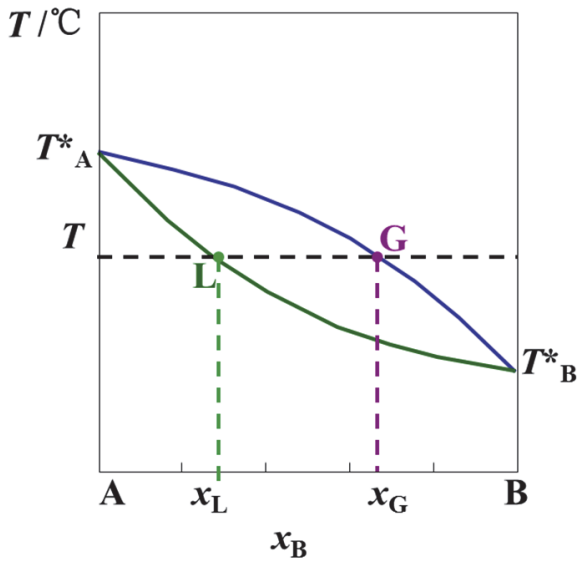

图 1 恒压条件下丙醇-乙醇(A-B)温度-组成曲线

\section{2 从理论中来, 到实践中去, 实验使理论更深刻}

为了更好地将理论教学和实验教学结合起来, 在近两年的教学过程中, 本人作为理论课教师特 意承担了两个小班的实验课教学任务。在实验课上, 教师会对本次实验课的实验原理以及理论课所 涉及的知识点进行提问, 帮助学生将课堂理论知识和实验原理结合起来。对于比较容易掌握的实验 原理, 鼓励学生在实验课上分组讲解实验原理, 提高学生实验课的参与度并有助于提高学生分析问 题和解决问题的能力。例如, 在 “最大气泡法测定溶液表面张力” 的实验中(图 2), 在实验原理的讲 解过程中再次强调, 测量的是压力最大值即液柱高柱的最大值; 而液柱高度最大, 恰恰是管口处气 泡曲率半径最小时的压力差。这一问题是本实验最容易混淆的地方, 也是在考试中出错率较高的考 点。在做实验的过程中再次强调这一问题, 可以加深学生的印象并对该知识点有深入理解。经实践, 关于最大起泡法知识点的问题在期末考试中, 正确率有明显的提升。再如: 在 “分配系数和平衡常 数的测定” 实验中, 课前提问本次实验的原理是什么? 学生通过理论课的学习根据分配定律知道分 配系数越大, 萃取效率越高, 那么在实验中是如何实现? 这就是我们本堂课要掌握的内容, 通过滴 定的方法测定分配系数。在实验课上简单的几分钟问答, 不仅提升了学生上实验课的积极性, 还可 
以引起学生对实验课的重视程度, 活跃课堂气氛, 更重要的是将理论和实践进行结合, 让学生们在 实验中对理论知识加深理解。在实验的过程中, 针对学生不清楚或者容易混淆的地方, 适当地予以 纠正和强调, 再次加深了学生对于知识点的认识和理解。

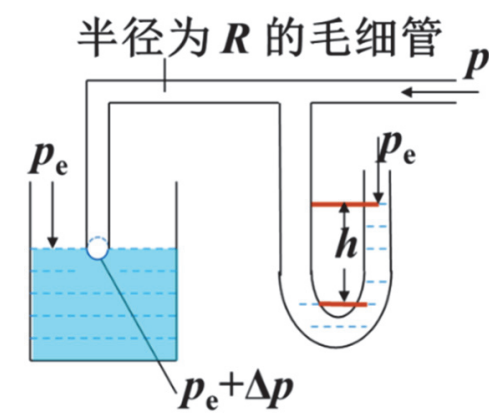

图 2 最大气泡法测量表面张力实验装置示意图

\section{3 强调实验结果的讨论与分析}

实验结果的分析与讨论是科学研究和实际生产中非常重要的一个组成部分 ${ }^{[7]}$ 。而在本科生的实 验教学过程中, 学生往往为了追求高分而主观降低实验误差, 追求实验结果 “完美”, 接近标准值。 为解决实验结果千篇一律、实验数据全班雷同的问题, 要求学生在实验结束时当场提交原始实验数 据, 并且说明数据没有好坏、对错之分, 实事求是是对待实验最根本的态度, 并逐步培养学生学会 并掌握误差产生的原因以及如何分析讨论实验结果, 这与理论知识的掌握程度是息息相关的。例如: 在 “蔗糖水解反应速率的测定” 实验中, 学生在实验过程中往往过度关注旋光度的绝对值, 会与其 他小组进行比较, 归其原因是对实验原理的理解不够清晰。在课堂上, 首先严格杜绝 “修改” 实验 数据的现象发生, 所有的原始数据必须经教师现场签字, 不得修改。学生在课后处理数据的过程中 会发现, 虽然不同组的实验绝对值会存在差距, 但是并不影响动力学曲线的绘制, 这是由于浓度的 计算是一个相对值, 需要减掉浓度的无穷值进行校正。引导学生认真对待数据处理和实验结果的讨 论与分析, 在对数据的分析和讨论过程中, 要结合实验操作、仪器等诸多影响因素, 找到影响数据 结果的关键因素和条件, 分析数据产生误差的原因。在课堂上不断地向学生灌输实验结果没有标准 答案, 在对实验结果的分析讨论中, 不仅要找到可能的操作失误, 还要找到哪一个是起决定作用的 影响因素, 只要分析讨论合理, 理由充分, 即使是误差再大的实验数据也可以得到优秀的实验成绩, 这样学生才能够不怕失败、实事求是, 逐步学会正确分析问题并真正提高解决问题的能力。

\section{4 与科学前沿结合, 鼓励学生参与研究实践}

知行统一, 理论联系实际, 用理论指导实践, 在科研实践结果中, 找到理论依据, 这一方法论, 无论对于教学还是科研都是十分重要的 ${ }^{[8-10]}$ 。本人所从事的科研项目中涉及到超浸润膜材料的制备 与应用。因此, 在理论课讲到 “界面现象” 一章时, 我会简单介绍超浸润材料的前沿进展并指出本 领域中存在的一些科学问题, 既开拓了学生的知识面, 又让学生理解学有所用, 对科学研究产生一 定的兴趣。在讲解什么是界面这一概念时, 我会列举生活中的一些实例来引入, 首先提问 “同学们 在日常生活中有没有注意到水滴在荷叶上是什么状态的？” 进而提问 “水滴在玫瑰花和水稻表面上 是什么形状的, 这三种植物表面与水滴接触的区别? ” 再进一步跟学生阐述三种界面虽然都是液体 与固体相接触, 且水滴在植物表面的形状类似, 但是运动状态是不一样的, 水滴在荷叶表面容易滚 落, 水滴在玫瑰花表面很难滚落, 水滴在水稻叶表面沿叶脉方向容易滚落, 这是什么原因呢, 其中 的科学道理就是界面现象这一章要讲述的问题。时间虽短, 但是却能激发学生学习的热情和对科学 研究的兴趣, 让学生感受到课堂上所学的理论知识是真正学有所用的。又如: 在 “电化学”一章中, 
受课时所限我们只有一节课的时间给学生介绍化学电池, 而这部分往往是学生感兴趣的内容, 但不 是物理化学教学大纲的重点。结合本校实际情况, 有些教师正开展前沿的锂电池、钠电池等科学研 究, 鼓励学生利用课余时间查阅书籍和文献, 并到科研实验室进行参观和学习, 全方位了解电池的 组装过程, 更有助于学生对电池原理的理解和消化。课堂的教学时间是有效的, 鼓励学生利用课余 时间参与科学研究, 尤其是与课堂知识相关的科学研究, 这不仅有利于学生掌握课堂知识, 更有助 于学生开拓眼界, 达到更高的高度。

\section{3 理论教学与实践教学互相渗透教学模式对教学效果的影响}

\section{1 知识点理解}

近两年，本人将理论教学与实践教学互相渗透的教学模式运用到物理化学课程的教学过程中, 已经取得了明显的教学效果。最突出的表现是在理论课和实验课中重复出现的知识点, 学生理解程 度明显改善, 作业以及考试相关问题的正确率显著提高。在以往的实验中, 大多数学生往往是为了 完成任务而做实验, 只进行简单机械的实验操作, 而近两年的学生在实验的预习及操作过程中, 能 够主动地发现问题, 积极思考, 并与老师进行讨论, 达到了实践教学与理论教学的良好互动。其次, 良好的教学效果也体现在实验报告中。学生不再追求 “完美数据” 的实验报告, 而是在对实验原理 充分理解的基础上, 对实验结果进行分析, 并对误差提出合理的解释。近两年学生实验成绩的优秀 率从原来的 $50 \%$ 左右提升到目前的 $70 \%$ 左右。

\section{2 自学能力}

大学教育不能仅仅是教师 “一言堂”, 大学阶段课程所涉及的知识点是非常繁多的, 远远超过 课堂上所能讲授的内容。“师傅领进门, 修行在个人”, 用这句话来形容大学生的学习状况是非常 恰当的。我们要培养的是具有专业知识和创新能力的全方位人才, 因此在理论和实践互相渗透的教 学模式中还应该尤为注重学生自主学习能力的培养。课堂时间永远是有限的, 无论对于理论课还是 实验课, 自学能力的培养是非常重要的。由于在理论课堂上对学生进行了充分的引导, 所以学生们 养成良好的预习习惯, 自学能力有所提高。在实验报告的完成过程中, 学生充分利用各类资源, 如 网络、图书馆等, 通过查阅资料和文献来解释相关的实验现象并扩充自己的知识面。例如在物理化 学实验结果和讨论部分, 教师不可能列举出所有可能出现的问题和产生误差的原因, 需要学生通过 自主调研文献来解决相关问题，自学能力和查阅资料、文献的能力大大加强。

\section{3 科研能力}

对于本专业的学生来说, 越来越多的学生本科毕业后选择考取研究生, 因此本科阶段科研能力 的提升也是非常重要的。而科研能力的培养往往就在于理论课和实验课的结合点。目前, 许多高校 都开展了各种形式的面向本科生的科技竞赛, 实际上就是鼓励本科生根据所学的知识, 能够有所创 新, 有所发现。本人曾指导由本科学生组成的研究小组开展 “静电纺丝聚合物膜利用双通道装置实 现可持续的油水分离” 项目参加学校科技竞赛并获奖。该项目涉及 “界面现象”一章中关于表面张 力的知识点, 这一课题既涉及到了物理化学中固体表面浸润性的问题, 又融入了一定的科研探索问 题, 是课堂知识点联系应用和实践的一个典型实例。学生在完成项目的过程中, 既对表面张力的概 念有了更深入的认识, 又对物理化学课程及科学研究产生了一定的兴趣, 受益匪浅。学生在教师的 指导下结合课堂所学知识, 通过文献调研, 自主设计实验方案, 更多地发挥主观能动性, 深化了课 堂上所学的知识点, 又为将来攻读研究生打下基础。本校十分重视本科生的科创项目, 约有 $50 \%$ 以 上的本科生会参加各类的科技创新活动, 随着目前各个高校科创项目逐渐增多, 会有更多的学生参 与到这类项目当中, 而且涉及的不仅仅是物理化学这门课程, 还有其他的专业课程, 因此将理论课 和实验课适当地融合起来, 对于学生综合能力的提升非常重要。 


\section{4 结语}

通过近几年在物理化学理论课和实验课教学过程中的实践与探索, 这种理论教学与实验教学互 相渗透的教学模式对于教学效果的提升是非常明显的。首先, 理论和实验互相渗透可以激发学生学 习的积极性, 调动学生自主学习的兴趣; 其次, 可以让教师和学生从枯燥的公式推导中走出来, 理 论联系实际, 让学生感受到理论知识如何解决实际问题; 再次, 在实验中发现规律, 找出原因, 对 于培养学生解决问题的能力和科研思维的方式非常重要。总之, 通过有意识地将理论知识与实验教 学结合起来, 可以使理论与实践相辅相成, 互相促进, 既活跃了课堂气氛又提升了教学效果, 值得 推荐。

\section{参 考 文 献}

[1] 张树永, 侯文华, 刀国旺. 大学化学, 2017, 32 (2), 9.

[2] 郭婷, 孟涛, 方伊, 童志平, 舒学涁. 实验室研究与探索, 2015, 34 (5), 38.

[3] 杨风霞, 安彩霞, 王爱荣. 化学教育, 2016, 37 (24), 16.

[4] 刘永梅, 张晋芬, 孙立森, 戴维林, 李晔飞. 大学化学, 2018, 33 (10), 18.

[5] 赵丽娜, 李欣, 任玉刚, 杨潇, 姜大雨. 实验技术与管理, 2015, 32 (3), 96.

[6] 庞秀江, 陈利, 赵继宽. 化学教育, 2008, No. 2, 39.

[7] 田福平, 贾翠英, 陈静, 贺民. 实验技术与管理, 2011, 28 (11), 109.

[8] 庄媛, 刘杰民, 姚喆. 大学化学, 2017, 32 (7), 44.

[9] 田东亮. 大学化学, 2018, 33 (3), 17.

[10] 胡新根, 何道法, 李新华, 方国勇, 王舜. 实验室研究与探索, 2009, 28 (10), 133. 